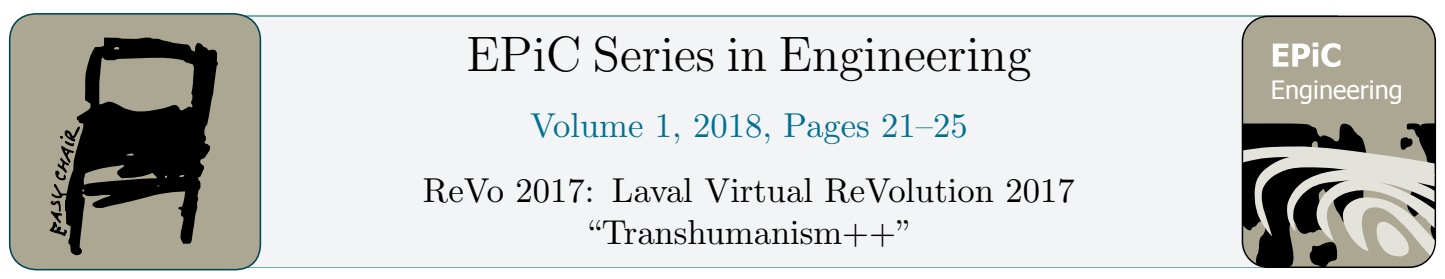

\title{
A High Immersive Infinity Walking System with HMD and Load Balance
}

\author{
Tomoyuki Yamamoto ${ }^{1}$, Yasuhiro Seya ${ }^{2}$, Mitsuyuki Saito ${ }^{1}$, \\ Yasuhide Kobayashi ${ }^{1}$, and Wataru Wakita ${ }^{1}$ \\ ${ }^{1}$ Hiroshima City University \\ ${ }^{2}$ Aichi Shukutoku University \\ wakita@hiroshima-cu.ac.jp
}

\begin{abstract}
We propose a low-cost and high immersive infinity walking system with HMD and all directional load balance, which enables to present walking sensation for a user in VR environments. By using a device supporting user's thighs during walking, our system enables a user to shift the center of gravity of body toward any walking direction like dynamic walking in real environments. Although many studies have proposed infinity walking systems based on a treadmill, a stepping method, a sliding method, and etc., there are several limitations to use these systems in practice. For example, the treadmill-based walking system is large and high-cost. The stepping method is known to be a low-cost method, but it is difficult for a user to shift the center of gravity of body toward a walking direction. The walking sensation provided by the sliding method may not be natural, because user's feet move backward during walking. Therefore, we develop a low-cost and high immersive infinity walking system with HMD and all directional load balance that can provide the realistic walking sensation with a user.
\end{abstract}

\section{High Immersive Infinity Walking System}

Many studies have proposed infinity walking methods for walking in virtual space. For example, walking on a treadmill enables a user to step and feel the sensation of natural walking. However, such system is high-cost system, because it is complexed structure such as its belt of torus structure. As a low-cost method, a stepping method [1] is proposed. This method enables a user to change a walking direction easily, but it is difficult to shift the center of gravity of body toward the walking direction. A sliding method in which a user wears a sliding device on the feet [2] also enables the user to step like the natural walking. However, walking sensation provided by this method may be relatively unrealistic because feet move in the opposite direction during walking. In general, the center of gravity of body shifts toward a walking direction, and our feet do not move backward while walking. Therefore, to 
develop a natural infinity walking system, it is very important to solve these points. Here, we propose a new walking platform that enables a user to shift the center of gravity of body toward any walking direction without sliding feet.

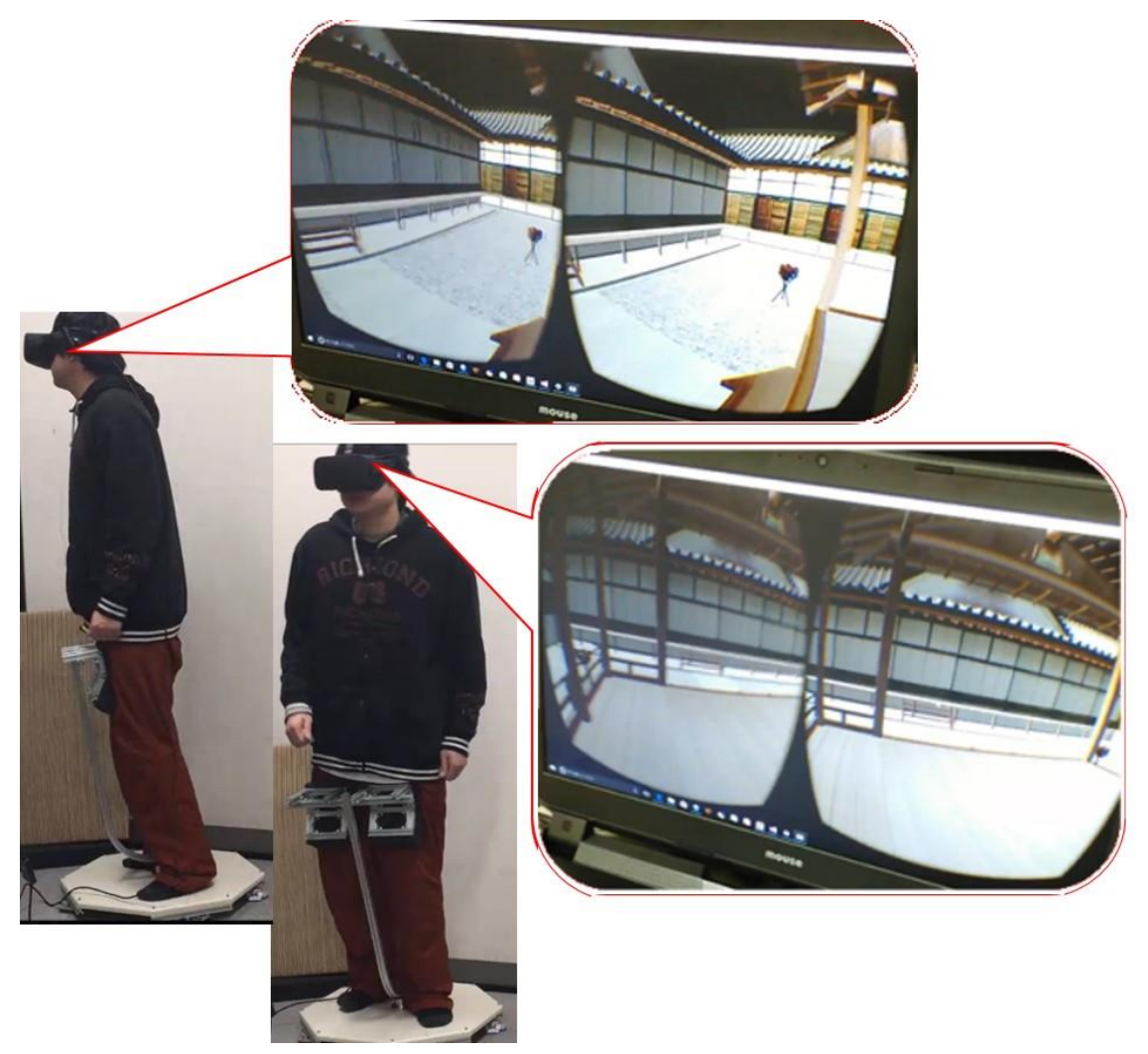

Figure 1: Proposal infinity walking system.

Figure 1 shows our proposal infinity walking system. Our system composes of a frame, sponge, bearing, potentiometer, HMD, and four force sensors (Figure 2). The frame plays an important role in supporting user's body leant toward a walking direction. The sponge is used to reduce feeling of pressure created with the contact of user's thighs and the frame. The bearing is used to rotate the frame in any walking direction. The potentiometer is used to measure walking direction. HMD is used to present visual image in VR environment. The force sensors are used to measure user's load balance toward user's anterior-posterior and left-right directions during stepping and leaning with propping user's thigh on our device. The walking speed is estimated from the load balance toward user's front direction. The visual image is changed, depending on user's walking motion and direction estimated with all directional load balance and the potentiometer. Thus, our system is expected to enable user to present the walking sensation with visual image and change the position of center of gravity of body toward walking direction such as dynamic walking in VR environment (Figure 3). In comparison with stepping method, our system enables user to change the balance toward walking direction. 


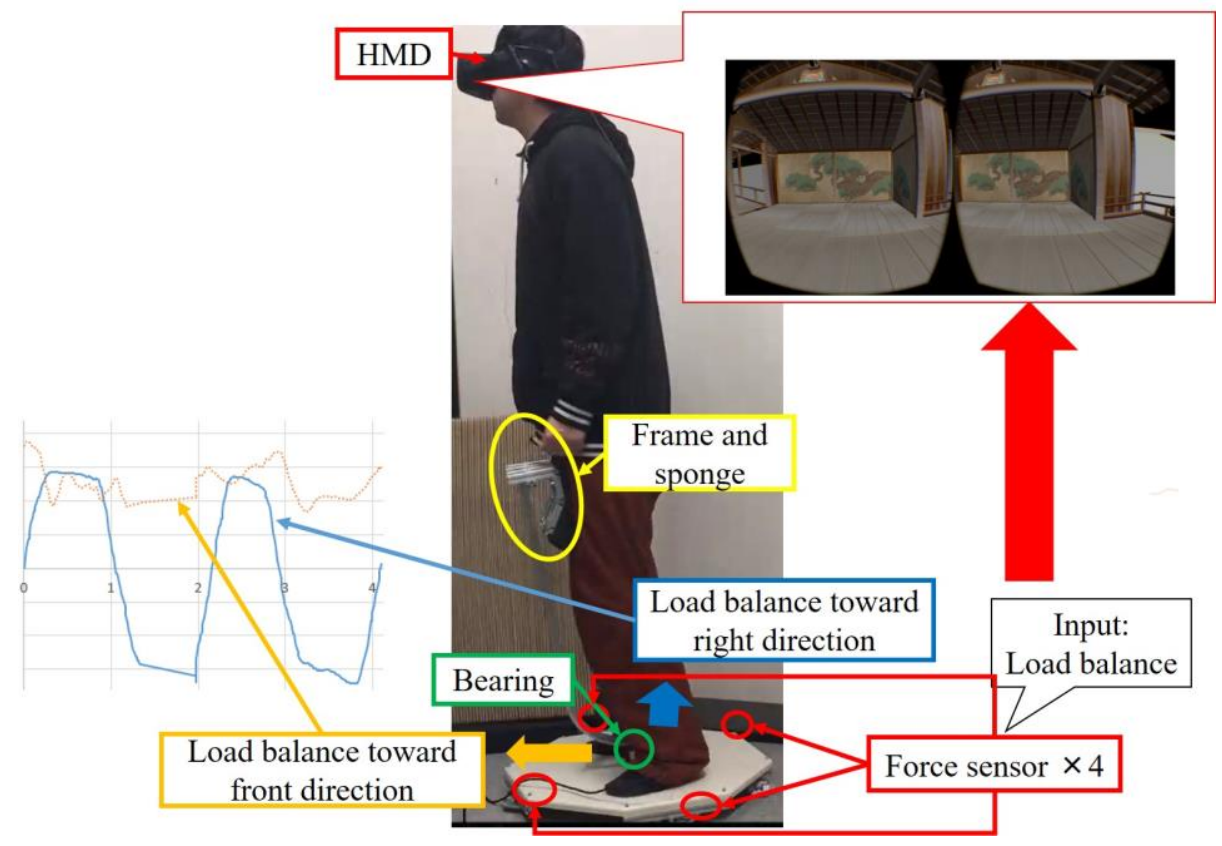

Figure 2: Overview of proposal infinity walking system.

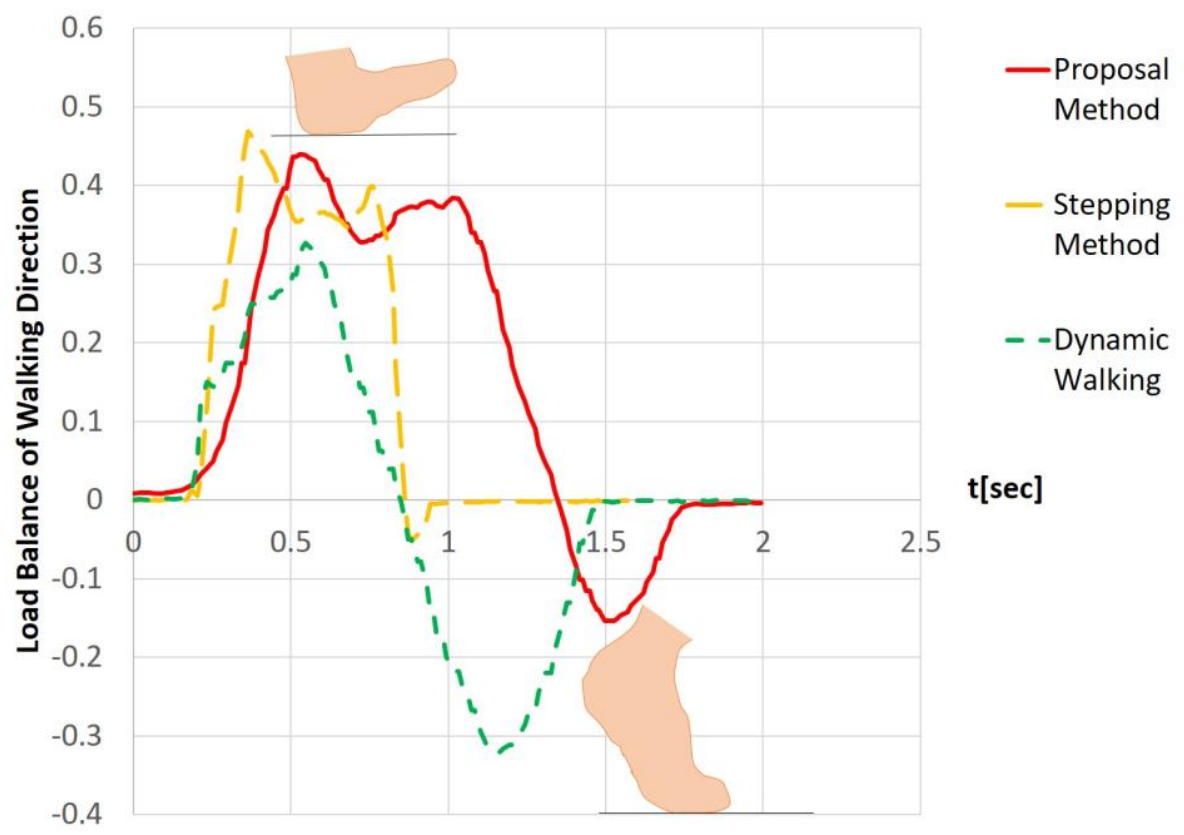

Figure 3: Result of change of the balance of center of gravity toward walking direction. 


\section{Load Balance}

Load balances are calculated with a force value $l f_{f}, l f_{b}, r f_{f}$, and $r f_{b}$ sensed by fore force sensors (Figure 4). $l b_{f}$ is regarded as load balance toward user's front, $l b_{r}$ is regarded as load balance toward user's right direction. $l b_{f}$ and $l b_{r}$ are given by:

$$
\begin{gathered}
f s u m=l f_{f}+l f_{b}+r f_{f}+r f_{b} \cdots(1) \\
l b_{f}=\frac{l f_{f}-l f_{b}+r f_{f}-r f_{b}}{f s u m} \cdots(2) \\
l b_{r}=\frac{l f_{f}+l f_{b}-r f_{f}-r f_{b}}{f s u m} \cdots(3)
\end{gathered}
$$

Moreover, load balances are changed with an affine transformation when user's walking direction is changed. $\theta$ sensed by the potentiometer is regarded as user's walking direction. The load balances $\widehat{l b}_{f}$ and $\widehat{l b_{r}}$, which are changed with an affine transformation, are given by:

$$
\begin{aligned}
& \widehat{l b_{f}}=l b_{r} \cos \theta-l b_{f} \sin \theta \cdots(4) \\
& \widehat{l b_{r}}=l b_{r} \sin \theta-l b_{f} \cos \theta \cdots(5)
\end{aligned}
$$

With (4) and (5) expressions, load balances are calculated toward all direction. Moreover, a threshold is set toward $\widehat{l b}_{f}$ and $\widehat{l b}_{r}$ because stepping motion is divided from standing state. Thf is regarded as threshold toward $\widehat{l b}_{f}$. Also, thr is regarded as threshold toward $\widehat{l b}_{r}$. When $\widehat{l b}_{f}$ is larger than or equal to thf and $\left|\widehat{l b}_{r}\right|$ is larger than or equal to thr, a user is walking motion on the device. When $\widehat{l b}_{f}$ is less than thf and $\left|\widehat{l b}_{r}\right|$ is less than thr, a user is standing state on the device. In this method, thf is 0.08 and thr is 0.25 .

A step length is necessary because a user walk in VR environment. $D$ is regarded as the step length. $D$ is given by:

$$
D=\operatorname{par}\left|l b_{f}\right| \cdots(6)
$$

Par is regarded as parameter for adjusting $D$. In this method, par is 0.25 .

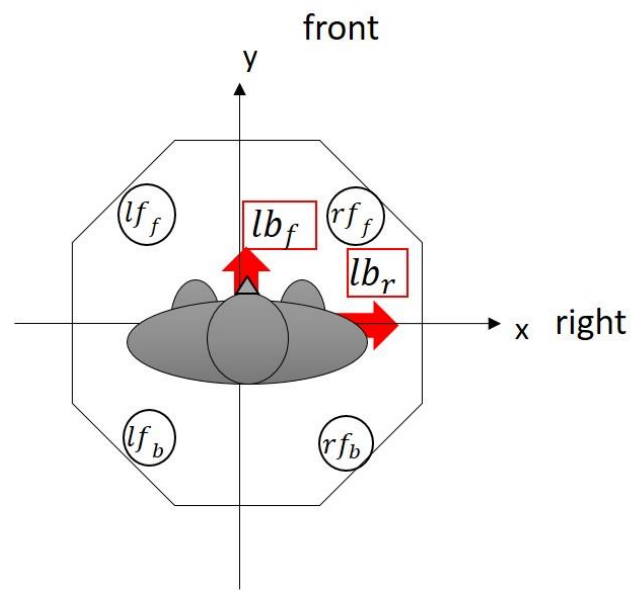

(a) Initial state

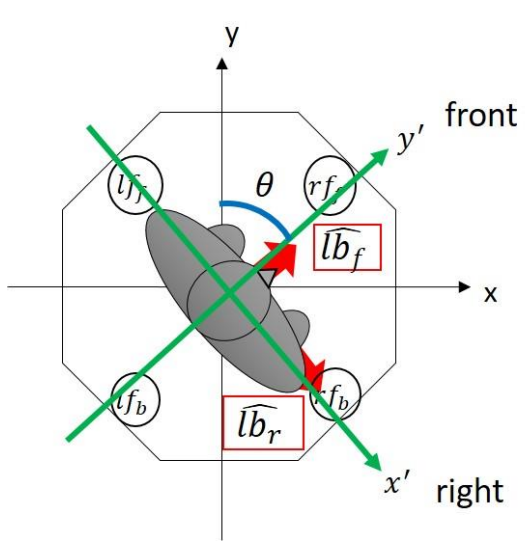

(b) After affine transformation

Figure 4: Load balance. 


\section{Conclusion and future work}

Our system enables a user to walk any forward direction in VR environments. However, our system cannot simulate walking backward and sideward. Moreover, our system enables a user to walk in flat surface only, not in uneven surface. Further studies should be necessary to solve these points.

\section{References}

[1] Sam Tregillus and Eelke Folmer. (2016). VR-STEP:Walking-in-Place using Inertial Sensing for Hands Free Navigation in Mobile VR Environments. Proc.CHI'16. pp.1250-1255.

[2] Iwata, H. and Fuji, T. (1996). VIRTUAL PERAMBULATOR: A Novel Interface Device for Locomotion in Virtual Environment. Proc IEEE VR 1996. pp. 60-65. 